\title{
GASTROPARESIA DIABÉTICA \\ Comparación del vaciamiento gástrico de pacientes tratados con eritromicina o cisaprida
}

\author{
Patricia Reyes M.D., William Rojas M.D.*
}

\section{Resumen}

En 1958 aparece el término gastroparesis diabeticorum para describir el proceso de atonía y alteración del vaciamiento gástrico, encontrados en algunos pacientes diabéticos. La prevalencia varía de 10 a $76 \%$, variación ocasionada por la poca especificidad de los síntomas. Se ha encontrado relación entre el adecuado control de glucemia y la absorción de medicamentos y el vaciamiento gástrico, por lo que es importante el tratamiento. Con este estudio se pretende comparar la eficacia clínica y paraclínica entre la eritromicina y el cisapride, utilizados aisladamente en gastroparesía. Se tomaron 18 pacientes diabéticos tipo 1 y 2 , con un año de evolución de la diabetes, edad media de 57.82 años, excluyendo pacientes con patología y medicamentos que alteraran el vaciamiento gástrico. Se elaboró un cuestionario sobre resultados antes y después del tratamiento y respecto al vaciamiento antes y después de quince días de tratamiento. Los síntomas más frecuentes fueron Ilenura posprandial, pérdida de peso, dispepsia y distensión abdominal. Nueve pacientes recibieron cisapride, ocho presentaron mejoría subjetiva, $55 \%$ mejoría del vaciamiento y en uno no cambió. Diez pacientes recibieron eritromicina, un diabético se retiró por intolerancia, siete presentaron mejoría subjetiva y $45 \%$ mejoría en los vaciamientos; $\left(X^{2}=1\right)$. No hubo diferencia significativa entre cisapride y eritromicina en la respuesta del vaciamiento gástrico. Rep Med Cir 2000 Nov; 9(2):8-12.

\section{Introducción}

En 1958, Kassander creó el térmico gastroparesis diabeticorum para describir el proceso de atonía y pérdida del vaciamiento gástrico observado en algunos pacientes diabéticos (1). Los diferentes métodos radiológicos o gammagráficos demuestran dilatación gástrica, disminución o ausencia de peristalsis, retención prolongada de bario, atonía del bulbo duodenal y retardo del vaciamiento gástrico.

Los síntomas gastrointestinales se presentan en pacientes con diabetes mellitus. No es una patología que se considere de alta morbilidad por lo que la mayoría de estudios lo considera de baja prevalencia, pero cuando se interroga específicamente sobre síntomas gastrointestinales esta se incrementa. La gastroparesía diabética se encuentra en 10-76\% de individuos con diabetes mellitus con ocho a diez años de evolución de la enfermedad (2).

Las manifestaciones de gastroparesia diabética son poco específicas; aproximadamente $50 \%$ de los pacientes son asintomáticos o coinciden con otros procesos patológicos gastrointestinales, esofagitis

\footnotetext{
* Servicio de Endocrinologia, Hospital de San José, Bogotá, Colombia
}

péptica, reflujo biliar, esofagitis candidiásica y bezoares, los cuales dificultan el diagnóstico. Los síntomas de gastroparesia diabética pueden ser náusea, vómito, distensión abdominal y anorexia (3). La importancia de instaurar tratamiento para la gastroparesía diabética consiste en la disminución de los efectos en el control de la glucemia (4), la absorción de medicamentos $(5,6)$ y la posibilidad de broncoaspiración (2).

Richard McCallum cuestionó el diagnóstico de gastroparesia diabética. Normalmente los alimentos sólidos inician su paso al duodeno a los $45 \mathrm{mi}$ nutos de su ingestión. En cambio, en la gastroparesía hay incremento del tono basal del esfínter pilórico con ausencia de las contracciones gástricas. Por ello, el método diagnóstico de elección para el estudio de la gastroparesia diabética es la utilización de un alimento sólido radiomarcado para lograr su visualización gammagráfica.

En el tratamiento farmacológico utilizado se encuentran agentes liberadores de acetilcolina, como cisapride, y agonistas del motilín como la eritromicina. El cisapride, derivado de la benzamida, estimula la motilidad antroduodenal al inducir la liberación de acetilcolina para actuar en los recepto- 
res muscarínicos. La eritromicina, antibiótico de tipo macrólido, estimula las contracciones de la fase III(7), al actuar como el motilin. Los receptores del motilin se encuentran en el antro gástrico y duodeno proximal $(6,8,9,10)$.

El propósito de esta investigación es comparar la eficacia clínica y paraclínica entre eritromicina y cisapride en pacientes con gastroparesía diabética.

\section{Materiales y métodos Pacientes}

El estudio se practicó en dieciocho (18) pacientes diabéticos tipo 1 y 2 , voluntarios, nueve hombres y nueve mujeres, que asistieron a la consulta de endocrinología de Hospital de San José, y cuya enfermedad tenía una evolución de más de 12 meses. Se excluyeron pacientes que estuvieran tomando cualquier medicación que afectara la motilidad gastrointestinal, como metoclopramida y domperidone; o presentaran procesos patológicos que intervinieran en el vaciamiento gástrico, como enfermedad de tejido conectivo, esclerodermia, anemia perniciosa, enfermedad neuromuscular, hipertiroidismo o historia de cirugía del tracto gastrointestinal superior.

\section{Protocolo}

Se elaboró un cuestionario para cada paciente a fin de evaluar los síntomas de gastroparesia diabética, antes y después de realizar vaciamiento gástrico para sólidos. Los 18 pacientes se clasificaron al azar en grupo $\mathrm{A}=$ grupo de pacientes que recibieron cisapride, $10 \mathrm{mg}$ quince minutos antes de cada comida, y grupo $\mathrm{B}=$ grupo de pacientes que recibieron eritromicina, $500 \mathrm{mg}$ quince minutos antes de cada comida. A ambos grupos se les administró medicación por mínimo quince días consecutivos, tiempo en el cual se les realizó otro cuestionario sobre síntomas de gastroparesia diabética y control de vaciamiento postratamiento.

\section{Evaluación de síntomas gastrointestinales}

El cuestionario para evaluar síntomas de gastroparesia diabética incluyó distensión abdominal, llenura posprandial, pérdida de peso, dispépsia, náuseas y vómito. Los síntomas eran graduados de acuerdo a los síntomas referidos en $\underline{\text { Nunca presenta sín- }}$ tomas, $\underline{\mathbf{A}}$ veces presenta síntomas (refiere síntomas una

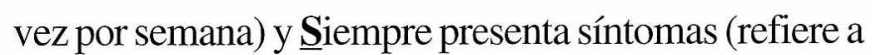
síntomas más de dos veces por semana) (Tabla). La mejoría subjetiva se evaluó al tener en cuenta la disminución de los síntomas.

Tabla

\begin{tabular}{|llll|}
\hline \multicolumn{4}{|c|}{ Evaluación de síntomas gastrointestinales } \\
\hline Síntomas & $\mathrm{N}$ & $\mathrm{A}$ & $\mathrm{S}$ \\
Distensión abdominal & 9 & 7 & 2 \\
Llenura posprandial & 6 & 9 & 3 \\
Pérdida de peso & 6 & 9 & 3 \\
Náuseas & 11 & 6 & 1 \\
Vómito & 16 & 2 & 0 \\
Dispepsia & 7 & 9 & 2 \\
\hline
\end{tabular}

$\mathrm{N}=$ Nunca; $\mathrm{A}=\mathrm{A}$ veces; $\mathrm{S}=$ Siempre

\section{Medición de vaciamiento gástrico}

El vaciamiento gástrico se evaluó administrando S.Coloide $99 \mathrm{mTc} 1 \mathrm{mCi}$ oral, marcando huevos revueltos y obteniendo imágenes estáticas del estómago en proyección anterior durante 3 horas. Se les midió el tiempo en que la radioactividad gástrica se redujo a la mitad. El vaciamiento gástrico se evaluó en ayunas.

\section{Análisis estadístico}

La información obtenida se analizó utilizando el programa Epi Info 6.04. Se utilizó estadística descriptiva para describir los grupos, y para las diferencias entre estos se utilizó la prueba de T de Student y la Chi cuadrado corregida para muestras pequeñas.

\section{Resultados}

Los 18 pacientes valorados en el estudio, se caracterizaron por: edad media de 57,82 años (rango entre 21 y 68 años); tiempo de evolución de diabetes entre 12 a 420 meses (media de 120 meses); tratamiento con sólo insulina cinco pacientes, sulfonilureas cinco pacientes, biguanidas un paciente y siete pacientes con terapia mixta.

Entre los síntomas referidos los más frecuentes fueron llenura posprandial y pérdida de peso $(66.6 \%)$, dispepsia (61\%), distensión abdominal $(50 \%)$, náuseas $(38,8 \%)$ y vómito $(11 \%)$. (Tabla 1$)$. De los 18 pacientes del estudio 15 refirieron mejoría subjetiva. El vaciamiento gástrico antes de cualquier tratamiento mostró una media de 115,66 minutos (rango entre 55 y 180 minutos) con una desviación estándar (DE) de 36, y el vaciamiento pos- 
tratamiento tenia una media de 108,66 minutos (rango entre 55 y 180 minutos) y una DE de 32,83 . Con una $p$ entre pre y postratamiento de 0.5 .

Grupo A o cisapride: Nueve pacientes fueron los que recibieron cisapride, con edad media de 61,5 años (39 - 79 años) y tiempo de evolución de diabetes entre 12 y 380 meses (media de 132,8 meses), de los cuales ocho refirieron mejoría subjetiva. El vaciamiento gástrico previo tratamiento era de 122,4 minutos (rango entre 70 y 180 minutos), DE $=37.7$. El vaciamiento pos-tratamiento disminuyó a 111,66 minutos (rango entre 70 y180 minutos), $\mathrm{DE}=38,2$. Para el análisis se excluyó el paciente que presentó vaciamiento gástrico mayor de 180 minutos por ser un solo caso, que sesgaría el resultado del grupo.

Excluyendo este paciente, el vaciamiento antes del tratamiento fue de 115,25 minutos (rango entre 70 170 minutos) $\mathrm{DE}=33,08$; y postratamiento fue de 103,25 minutos (rango entre 70 y 155 minutos). Comparando los vaciamientos $55,5 \%$ de los pacientes presentaron mejoría, 33,3\% empeoraron y en un paciente (vaciamiento gástrico mayor de $180 \mathrm{mi}-$ nutos) no hubo cambio.

Grupo B o eritromicina: A diez pacientes se les inició eritromicina, pero un paciente presentó intolerancia a la segunda dosis de la medicación por lo que se retiró de este grupo y se pasó al grupo A, quedando nueve pacientes que recibieron eritromicina, con edades que variaban entre 21 y 79 años (media de 54,11 años) y tiempo de evolución de diabetes de 158,66 meses (rango entre 18 y 420 meses), de los cuales siete presentaron mejoría subjetiva.

El vaciamiento gástrico previo tratamiento fue de 108,88 minutos (rango de 55 a 160 minutos), $\mathrm{DS}=35,06$, y el post-tratamiento disminuyó a 105,66 minutos (rango de 75 a 150 minutos), $\mathrm{DE}=28,42$. Comparando los vaciamientos, $45 \%$ de los pacientes presentaron mejoría, $55 \%$ empeoraron.

$\mathrm{Al}$ comparar la respuesta entre los dos medicamentos se encontró $\mathrm{X}^{2}$ corregido de Yates $=1$.

En la mayoría de los pacientes de la investigación, no se comprobaron efectos secundarios con cisapride o eritromicina. Sin embargo, dos pacientes con eritromicina refirieron flatulencia, náuseas y distensión abdominal, por lo cual a uno se le suspendió eritromicina con la segunda dosis y se le inició cisapride, que toleró satisfactoriamente.

\section{Discusión}

Nuestro estudió de 18 pacientes no demostró diferencia en el vaciamiento gástrico de pacientes con gastroparesia diabética, que recibieron tratamiento con eritromicina o cisapride. Sin embargo, clínicamente, el grupo que recibió cisapride mostró leve mejoría subjetiva al compararlo con el tratado con eritromicina.

La mayoría de los síntomas manifestados por los pacientes fueron clasificados como "a veces", y muy pocos expresaron severas manifestaciones gastrointestinales. Encontrar pacientes asíntomáticos nos permite llamar a este fenómeno "gastroparesia silente", y el hallar discordancia entre los síntomas de gastroparesia y vaciamiento gástrico ocasiona dificultad en el diagnóstico presuntivo de gastroparesia diabética(11).

La gastroparesía silente puede considerarse en los pacientes diabéticos como un análogo de infarto del miocardio silente. Con la introducción de este nuevo concepto de "silente" o síntomas leves, Enck y Frieling proponen propiedades intrínsecas en la gastroparesia diabética que requieren atención específica en el diagnóstico y tratamiento. Una hipótesis, la de encontrar pacientes sintomáticos con vaciamiento normal, supone que hay incremento de la visceropercepción, secundario a los efectos directos de la hiperglucemia. Otra considera que, con la edad, los pacientes presentan mayor sintomatología gastrointestinal. Por eso, la mayoría de los pacientes que presentan gastroparesia silente son diabéticos tipo 1.

El compromiso de la actividad mioeléctrica gástrica está disminuida en diabéticos con neuropatia autonómica, y esta denervación autonómica se asocia con episodios de taquigastria, durante el cual se inhibe el vaciamieno gástrico acompañado de náuseas y/o vómito (11). La mayoría de los estudios sustentan un defecto de inervación por el vago, causante de las anormalidades de la motilidad gastrointestinal, aunque el estudio microscópico no ha mostrado cambios morfológicos del mismo (12).

El apropiado vaciamiento gástrico depende del equilibrio entre dos fuerzas: propulsivas (tono fúndico, contractilidad antral, coordinación antroduodenal) y de retención (contracción pilórica) (13). Alguna alteración del equilibrio de estas fuerzas ocasiona diferentes patrones de motilidad gastrointestinal. En los pacientes diabéticos se en- 
cuentra compromiso de las fuerzas propulsoras (menor número de contracciones), disminución de la relajación gástrica, con incremento de la contractilidad (movimiento retrógrado), incoordinación de la motilidad antroduodenal y movimiento transpilórico (14). Estos efectos ocasionan disminución del número de bolos que pasan a través de la unión gastroduodenal mientras que los movimientos retrógrados producen el reflujo del quimo del yeyuno al duodeno, ocasionando la gastroparesia.

La estrategia apropiada para tratar la gastroparesia diabética es al de buscar fármacos que actúan localmente sobre el sistema nervioso entérico al inducir diferentes patrones de motilidad gastrointestinal y aumentar la coordinación antroduodenal $(10,11,15,16)$. Esta acción directa explicaría porque en algunas circunstancias la eritromicina mejora el vaciamiento gástrico. El efecto de la eritromicina a nivel del esfínter esofágico inferior puede ser bloqueada por atropina lo cual sugiere que esta mediada por el vago (17). En conejos, se demostró que las contracciones son inhibidas por nifedipina; esto indica que la acción de la eritromicina es calciodependiente (17). En estado de ayuno bajas dosis de eritromicina disminuyen el efecto de motilin en la motilidad intestinal, al inhibir la unión a su receptor $(9,15)$. En estudios que valoran la concentración del polipéptido pancreático, este se incrementa con la eritromicina, por lo que interviene en la regulación del apetito e ingesta de comidas (16).

Aunque en nuestro estudio la mejoría con eritromicina no fue significativa, existen otros estudios que demuestran lo contrario. Richards y cols. demostraron mejoría significativa (39 y $60 \%$ respectivamente) utilizando eritromicina oral o endovenosa (18), y su mayor acción ocurre en los primeros 30 minutos pos infusión de eritromicina $(19,20)$. En nuestro estudio, la mayoría de los pacientes toleró apropiadamente las medicaciones, hubo dos pacientes del protocolo que no toleraron en forma adecuada la eritromicina, y uno no recibió la medicación, por presentar manifestaciones gastrointestinales. Revisando la literatura, se ha encontrado que algunos pacientes (7-10\%) presentan síntomas gastrointestinales, como náusea, vómito y diarrea (17).

\section{Conclusiones}

La gastroparesia diabética, como parte del complejo de complicaciones de la neuropatìa diabética, es una complicación a la que no se le da la suficiente importancia, y por tanto no se diagnostica tempranamente. No haber encontrado diferencia significativa paraclínica en el manejo con eritromicina o cisapride plantea la posibilidad terapéutica de la eritromicina para el tratamiento de la gastroparesia teniendo en cuenta que no seria una droga de primera elección, ya que en nuestro estudio encontramos que la tolerancia al medicamento fue menor que la mostrada por la cisaprida. Sin embargo, puede ser considerada una alternativa terapéutica util si tenemos en cuenta las complicaciones de la cisaprida (alteraciones de la conducción cardiaca) que en este momento están limitando su empleo.

\section{Referencias}

1. Yang R, Arem R, Chan L. Gastrointestinal tract complications of diabetes mellitus. Arch Intern Med. 1984; 144: 1251-6.

2. Zachary T Bloomgarden. American Diabetes Association Scientific Sessions. Diabetes Care. 1995; 18(9): 1314-8.

3. Vinik A I y Suwanwalaikorn. Autonomic Neuropathy. En: DeFronzo R. Current therapy of diabetes mellitus:. St Louis: Mosby; 1998.

4. Masataka I, Nakamura T, Kasai F, Onuma T, Bara T. y Takebe K. Altered postprandial Insulin requirement in IDDM patients with gastroparesis. Diabetes Care. 1994; 17: 901-3.

5. Jones KL, Horowitz M, Wishart JM, Maddox AF, Harding PE y Chatterton BE. Relationships between gastric emptying intragastric meal distribution and blood glucose concentrations in diabetes mellitus. J Nucl Med. 1995; 36(12): 2220-8.

6. Ishii Ma, Nakamura T, Kasai F, Baba T, Takebe K. Erytromycin derivate improves gastric emptying and insulin requirement in diabetic patients with gastroparesis. Diabetes Care. 1997; 20: 1134-7.

7. Tack J, Janssens J, Vantrappen G, et al. Effect of erithromycin on gastric motility in controls and in diabetic gastroparesis. Gastroenterology. 1992; 103: 72-9.

8. Soergel KH y Greenberger NJ. Nausea and vomiting in the diabetic patient. Hospital practice. 1998; 15: 43-62.

9. Peeeters TL, Muls E, Janssens J, et al. Effect of motilin on gastric emptying in patients with diabetic gastroparesis. Gastroenterology. 1992; 102: 97-101.

10. Parkman H, Pagano A P, Ryan J P. Erythromycin inhibits Rabbit pyloric smooth rmuscle through neuronal motilin receptors, Gastroenterology. 1996; 111: 682-90.

11. Enck P, Frieling T. Pathophysiology of diabetic gastroparesis. Diabetes. 1997; 46 S: S77-S81.

12. Nowak TV, Johnson CP, Kalbfleisch JH, et al. Highly variable gastric emptying in patients with insulin dependent diabetes mellitus. Gut. 1995; 37:23-9.

13. Lipp RW, Schnedl WJ, Hammer HF, Kotanko P, Leb G, Krejs GJ. Evidence of accelerated gastric emptying in longstanding diabetic patients after ingestion of a semisolid meal. J Nucl Med. 1997; 38: 814-8. 
14. Nguyen HN, Silny J, Wüller, Marschall HU, Rau G, Matern $\mathrm{S}$. Abnormal postprandial duodenal chyme transport in patients with long standing insulin dependent diabetes mellitus. Gut. 1997; 41: 624-31.

15. Annese V, Janssens J, Vantrappen G, et al. Erythromycin accellerates gastric emptying by inducing antral contractions and improved gastroduodenal coordination. Gastroenterology. 1992; 102: 823-8.

16. Stacher G, Peeters TL, Bergmann H, et al. Erythromycin effects on gastric emptying, antral motility and plasma motilin and pancreatic polypeptide concentrations in anorexia nervosa. Gut. 1993; 34:166-72.

17. Catnach SM, Fairclough PD. Erythromycin and the gut. Gut. 1992;33:397-401.
18. Richards RD, Davenport K, McCallum RW. The treatment of idiopathic and diabetic gastroparesis with acute intravenous and chronic oral erythromycin. Am J Gastroenterology. 1993; 88:203-11.

19. Keshavarzian A, Isaac RM. Erythromycin accelerates gastric emptying of indigestible solids and transpyloric migration of the tip of an enteral feeding tube in fasting and fed states. Am J Gastroenterology. 1993;88: 193-7.

20. Lin HC, Sanders SL, Gu YG, Doty JE. Erythromycin accelerates solid emptying at the expense of gastric sieving. Dig Dis Sci. 1994; 39(1): 124-8.

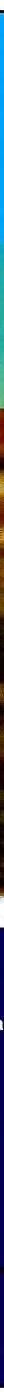

